Anke Berghaus-Sprengel

\title{
Forschungsnahe Dienstleistungen an der Universitäts- und Landesbibliothek Sachsen-Anhalt
}

\section{Ausgangslage und Schwerpunktsetzungen}

Die Universitäts- und Landesbibliothek Sachsen-Anhalt (ULB) versorgt die Martin-Luther-Universität Halle-Wittenberg (MLU) mit bibliothekarischen Dienstleistungen und trägt als Landesbibliothek die Verantwortung für das sachsen-anhaltische Pflichtexemplar sowie die Betreuung wertvoller Altbestände. Ein Schwerpunkt in den letzten Jahren lag auf der Standortkonzentration, d. h. mit der Reduzierung der Anzahl kleinerer Bibliotheksstandorte zugunsten großer, leistungsfähiger Bereichsbibliotheken wurde die Versorgung der universitären Nutzer räumlich neu aufgestellt, ohne jedoch das Dienstleistungsangebot grundsätzlich infrage zu stellen. Den weiteren Schwerpunkt bildete die Digitalisierung der Altbestände. Halle war wesentlich beteiligt an der Konzeption des VD18-Projekts, der Digitalisierung des deutschsprachigen Schrifttums des 18. Jahrhunderts. Mit der Digitalisierung von Drucken des 16. Jahrhunderts, der Mainstream-Literatur des 17. Jahrhunderts und der Drucke des 18. Jahrhunderts sowie zahlreichen weiteren Digitalisierungsprojekten wurden bis heute über 90000 Titel mit mehreren Millionen Seiten digitalisiert, persistent adressiert und im Open Access zur Verfügung gestellt.

Von ihren nur 113 Mitarbeiterinnen und Mitarbeitern, welche die verbliebenen 14 Standorte betreiben, konnten neben der Sicherstellung des Routinebetriebs nicht zeitgleich weitere Projekte oder Weichenstellungen begonnen werden. Mit dem Weggang der Leitung und der geschäftsführenden Besetzung der Vakanz in den Jahren 2013-2016 gab es keine Grundlage für eine systematische Weiterentwicklung der Dienstleistungen der Bibliothek. Erst nach der regulären Besetzung der Bibliotheksleitung im April 2016 konnten daher neue Schwerpunkte gesetzt werden.

Zuallererst waren die Rahmenbedingungen zu schaffen, die es den Mitarbeitern erlaubten, neben dem Alltagsgeschäft auch Zeit für neue Projekte erübrigen zu können. Bisher hatte z. B. die Einstellung studentischer Hilfskräfte darum viel Zeit gebunden, weil Verträge nur jeweils für ein halbes Jahr bewilligt worden waren. Die Universitätsleitung war bereit, die ULB dahingehend zu unterstützen, dass die Mittel ab 2016 für einen Zeitraum von je zwei Jahren bereit- 
gestellt wurden, sodass längerfristige Verträge möglich wurden. Die gesparte Zeit wurde durch die Erhöhung des Erwerbungsetats dann jedoch unmittelbar wieder in das Alltagsgeschäft investiert. Der Erwerbungsetat stagnierte seit zehn Jahren und reichte nicht aus, um den Bedarf an aktueller Literatur und Lizenzen zu decken. Die Universitätsleitung stellte daher der ULB einen um je 1,9 Millionen Euro erhöhten Etat für drei Jahre zur Verfügung. Das erhöhte Bestellvolumen band und bindet aber auch Kapazitäten, die an anderer Stelle nicht zur Verfügung stehen.

Verschärfend kam hinzu, dass seit 2016 über 50 \% der Abteilungsleiterstellen und einige Fachreferentenstellen neu zu besetzen waren. Aufgrund der Schwierigkeit, gut ausgebildetes IT-Personal und erfahrene Abteilungsleiter für die Betreuung historischer Sammlungen nach Halle abzuwerben bzw. auf dem Stellenmarkt zu finden, dauerten die Besetzungen häufig länger als beabsichtigt. Es kam oft genug vor, dass eine Neueinstellung auf einer der ausgeschriebenen Positionen eine Lücke an anderer Stelle gerissen hat. Die IT-Abteilung war mit wechselndem Personal so dünn besetzt, dass neben dem Betrieb des Bibliothekssystems Halle-Merseburg und der Betreuung der vorhandenen Arbeitsplatzrechner keine Kapazität für den Aufbau einer adäquaten IT-Infrastruktur vorhanden war. Die Rechner der Mitarbeiterinnen und Mitarbeiter waren als Einzelplatzrechner „stand alone“ eingerichtet und zum Teil 14 Jahre alt. Es gab keine zentrale Administration, keine Standardinstallationen und viele individuelle Softwarelösungen. Ausstehend war der Wechsel des Bibliotheksmanagementsystems LBS3 auf LBS4, den die ULB für die ULB SachsenAnhalt und die zehn weiteren Bibliotheken des Bibliothekssystems Halle-Merseburg 2016 mit dem OUS begann und mit der Umstellung des Erwerbungsmoduls ACQ Anfang 2017 abschließen konnte. Diese Basisdienstleistungen wurden erfolgreich erbracht, darüber hinausgehende Dienstleistungsangebote etwa im Bereich der forschungsnahen Dienstleistungen konnten erst aufgebaut werden, nachdem zusätzliches Personal in der IT zur Verfügung stand.

Die Bibliothek stand unter Handlungsdruck. Die Wissenschaftler benötigten für die Beantragung von Drittmittelprojekten die Zusage, dass deren Forschungsdaten sicher gespeichert werden konnten. Regelmäßig gab es Anfragen, ob nicht die Publikation im Open Access durch die Übernahme der Article Processing Charges unterstützt werden könnte, und die Bibliotheken in SachsenAnhalt mahnten erweiterte Funktionen des seit Langem existierenden, aber nicht weiterentwickelten Landesservers an. Dieser stand allen Bibliotheken im Land zur Ablage von Publikationen (Abschlussarbeiten, Dissertationen etc.) offen, verfügte jedoch nicht über Funktionalitäten zur Verwaltung heterogener Publikationen. Auch der 2016 bewilligte DFG-Fachinformationsdienst Nahost-, 
Nordafrika- und Islamstudien benötigte für den Ausbau seiner digitalen Angebote neue Infrastrukturen. ${ }^{1}$

Somit entschieden wir, dass die nächsten zu besetzenden Stellen im höheren Dienst zur Unterstützung der Open-Science-Aktivitäten genutzt werden sollten. Glücklicherweise war sowohl in der Universität als auch bei einzelnen Fachreferenten der Bibliothek die Bereitschaft groß, an der Umsetzung einer Open-Access-Strategie mitzuarbeiten. Ein erster Versuch, eine aus Stellenkürzungen erwirtschaftete befristete Stelle für die Einstellung eines Forschungsdatenbeauftragten einsetzen zu können, scheiterte daran, dass die guten Interessenten nur für eine unbefristete Stelle nach Halle wechseln wollten. Letztlich gelang es erst im ersten Quartal 2017, ein aktives und arbeitsfähiges OpenScience-Team zu haben. Das Team entwickelte ein umfangreiches Programm für 2017-2018 und setzte alle geplanten Meilensteine bisher erfolgreich um.

Mit der zunehmenden Bedeutung elektronischer Ressourcen war es notwendig, Rechercheangebote zur Verfügung zu stellen, mit denen unsere Repositorien ebenso durchsucht werden können wie auch die elektronischen lizenzierten Inhalte auf Artikelebene. Im Ergebnis einer Ausschreibung entschied sich die Bibliothek für EDS der Firma Ebsco und wird Anfang 2018 das neue Discovery-System produktiv schalten. Da die ULB Sachsen-Anhalt als Landesbibliothek und als Betreuerin des Bibliothekssystems Halle-Merseburg im GBV ${ }^{2}$ die Aufgabe hat, die Bibliotheken im Land bei der Bereitstellung von Dienstleistungen zu unterstützen, werden sowohl das Discovery-System als auch das Forschungsdaten- und Open-Access-Repositorium von den Bibliotheken genutzt werden können, um eigene Angebote auszubauen und zu profilieren.

Im Folgenden wird ausgeführt, welche Angebote und Aktivitäten im Bereich forschungsnaher Dienstleistungen im letzten Jahr durch das Team geplant und umgesetzt worden sind und was in diesem Jahr noch zu erwarten ist.

\section{Open Access}

Die ULB stimmt ihre strategischen Ziele mit den Wissenschaftlerinnen und Wissenschaftlern der Universität in der Bibliothekskommission ab. Es handelt sich dabei um eine Rektoratskommission unter Leitung des Rektors der MLU, die jährlich die Etatverteilung für den Literaturerwerb beschließt und in der die Bibliothek Rechenschaft ablegt. In der Kommission wurde zum Beispiel 2016 ent-

1 Vgl. http://bibliothek.uni-halle.de/projekte/fid_nahost/. Alle aufgeführten Internetressourcen wurden zuletzt am 2. Januar 2018 aufgerufen.

2 Gemeinsamer Bibliotheksverbund GBV: http://hhal.gbv.de/cgi-bin/wwwopc4menu. 
schieden, die DEAL-Initiative ${ }^{3}$ zu unterstützen. Die Initiative hat zum Ziel, bundesweite Lizenzverträge mit den großen Wissenschaftsverlagen abzuschließen. Die Verträge sollen so ausgestaltet werden, dass die Transformation von der subskriptionsbasierten Lizenzierung zu einer Finanzierung der Publikationen, die dann Open Access zur Verfügung stehen, befördert wird. Der Subskriptionsvertrag der ULB Sachsen-Anhalt mit Elsevier lief Ende 2016 aus und wurde nicht verlängert, eine Sperrung des Zugangs in Kauf genommen. Diese Entscheidung beschloss die Kommission einstimmig, obwohl mehrere Hunderttausend Zugriffe allein auf die Freedom Collection eine gute Nutzung attestierten. Neben der Bibliothekskommission wurde unter Leitung des Prorektors für Forschung vom Rektorat Ende 2016 eine Open-Access-Kommission eingesetzt, welche die Strategie der Universität in Bezug auf Open Access erarbeiten sollte. Diese Kommission setzt sich aus Mitarbeitern der Bibliothek und Wissenschaftlern verschiedener Fakultäten zusammen.

Die Kommission arbeitete zügig und legte dem Akademischen Senat bereits im Januar 2017 einen Entwurf für eine Open-Access-Policy der MLU zur Entscheidung vor. Die Policy ${ }^{4}$ setzt auf Freiwilligkeit und auf Unterstützung bei Open-Access-Publikationen. Publikationen im Gold Open Access werden durch einen Publikationszuschuss bis 2000 Euro gefördert. Die Entscheidung über die Gewährung des Zuschusses erfolgt nach DFG-Kriterien. Der Geschäftsgang ist schlank, die Beantragung unkompliziert online möglich. Finanziert wurde der Fonds im Jahr 2016 durch einen Teil der eingesparten Elsevier-Gelder. Den Hinweisen aus den Geisteswissenschaften, dass es gut wäre, auch andere Publikationskulturen zu fördern, wurde mit der Schaffung eines offenen Projektfonds entsprochen. Über diesen Fonds können ebenfalls bis zu einer Höhe von 2000 Euro Fördermittel beantragt werden, um Open Access zu publizieren. Es kann sich bei den Projekten sowohl um die Unterstützung der Veröffentlichung von Monografien handeln als auch um Layoutzuschüsse für die Gestaltung einer Open-Access-Zeitschrift oder um andere Projekte, die die Publikation im Gold Open Access beabsichtigen.

Bekannt gemacht werden die Angebote in Schulungen oder in kurzen „Open Science Lunch Breaks“, bei denen Antragstellung und Procedere sowie allgemein Publikationsberatung vermittelt werden. Im Frühjahr 2016 beantragte die ULB DFG-Mittel zur Publikationsförderung und bekam diese Ende 2017 genehmigt, sodass ab 2018 mit diesen Mitteln eine größere Anzahl von Anträgen bewilligt werden kann.

3 https://www.projekt-deal.de/.

4 Vgl. http://bibliothek.uni-halle.de/dbib/openaccess/. 
Neben der Publikationsförderung und -beratung wird das Hosting von Open-Access-Zeitschriften über ein Open Journal System (OJS) zur Verfügung gestellt. In Arbeitsteilung mit dem LLZ-Zentrum für multimediales Lehren und Lernen stellt die ULB den Server bereit und berät bei Format- und ErschlieBungsfragen, und das LLZ schult die Erstellung von Zeitschriften und die Veröffentlichung von Artikeln in OJS. Noch 2017 wurde beschlossen, den Server zu aktualisieren, aber die grundlegende Modernisierung wird erst 2018 beendet sein. Dann stehen verbesserte Workflows und andere Designoptionen zur Verfügung.

Ab 2018 soll ebenfalls die Möglichkeit bestehen, Zweitveröffentlichungen auf dem neuen Osiris-Server ${ }^{5} \mathrm{zu}$ publizieren. Dazu sollen möglichst viele der Publikationen, die an der MLU entstanden und die in der Hochschulbibliografie verzeichnet sind, nach Ablauf der Embargofrist auf dem Server publiziert werden. Das neue Repositorium löst den aktuell betriebenen Landesserver ab. Momentan publizieren dort die Hochschulen des Landes im Wesentlichen die Qualifikationsschriften ihrer Hochschulangehörigen. Geplant ist der automatische Versand einer Nachricht an die Autoren bei Einstellung der Artikel in die Hochschulbibliografie. In der Nachricht wird um Freigabe der Publikation für die Zweitveröffentlichung auf dem Osiris-Server gebeten. Die Veröffentlichungen können nach dem neuen Zweitveröffentlichungsgesetz für deutsche Publikationen sechs Monate nach Erscheinungsdatum eingestellt werden, wenn bis dahin die Autorengenehmigung vorliegt oder die Autoren selber ihre Publikation hochladen. Andere Fristen gelten bei internationalen Publikationen, die nach Zusage des Autors oder der Autoren individuell auf Wiedervorlage nach Embargofrist eingestellt werden müssen.

Osiris kann und soll aber weitere Dienste anbieten und dient dazu, Forschungsdaten verschiedener Art nachhaltig zu sichern.

\section{Forschungsdatenmanagement an der MLU}

Osiris ist eine Plattform, die hochschulintern zur Aufnahme von Zweitveröffentlichungen, Qualifikationsarbeiten, hochschuleigenen Open-Access-Publikationen, an der Hochschule herausgegebenen Open-Access-Zeitschriften, Forschungsdaten und Forschungsprimärdaten dienen soll. Zugleich wird das Repositorium auch außerhalb der MLU von anderen Hochschulbibliotheken genutzt werden können. Der neue Landesserver Osiris wird den verschiedenen Hoch-

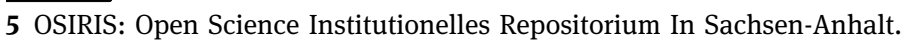


schulen Sachsen-Anhalts eine Ad-hoc-Lösung zur Adressierung ihrer publikations- und forschungsspezifischen Bedarfe zur Verfügung stellen. Es wird ein mandantenfähiges Datenrepositorium aufgebaut, das im Rahmen von Open Access und Open Science die MLU und die anderen Hochschulen Sachsen-Anhalts bei der Erfüllung ihrer Datenmanagement-, Publikations- und Datenaustauschverpflichtungen unterstützt.

Andere Hochschulen Sachsen-Anhalts bzw. nichtuniversitäre wissenschaftliche Bibliotheken können in eigenen Bereichen ihre elektronischen Abschlussarbeiten, Dissertationen, Open-Access-Publikationen oder Forschungsdokumente hochladen, Wissenschaftler ihre Publikationen selber einstellen. Die Freischaltung erfolgt nach Ergänzung der Metadaten durch die Bibliothek. Die automatische Vergabe von Identifiern und die DINI-Zertifizierung sichern die technisch-wirtschaftliche Nachhaltigkeit des aufzubauenden Serviceangebots.

Begleitet werden soll dieser Service von Schulungsangeboten für andere Hochschulen und Universitäten in Sachsen-Anhalt, damit vor Ort die kompetente Nutzung realisiert werden kann und die Bekanntheit im Land erweitert wird. So wie die Mitarbeiter anderer institutioneller Nutzer des Repositoriums geschult werden, so werden auch gezielt Schulungs- und Publikationslehrgänge für angehende Wissenschaftler und Professoren in Forschung und Lehre angeboten. An der ULB ist die Bereitstellung des Repositoriums und die Etablierung des Rechte- und Rollenkonzepts Teil der vielfältigen Aktivitäten zur Förderung von Open-Access-Angeboten an den Universitäten und Hochschulen im Land Sachsen-Anhalt.

Basis von Osiris ist eine D-Space-Installation, die dem Nutzer einen Einstiegspunkt bietet, um verschiedene Datentypen zu finden, $\mathrm{zu}$ verwalten und zugänglich zu halten. ${ }^{6}$ D-Space-Installationen stellen eine hohe Flexibilität bei der Veröffentlichung unterschiedlicher Publikationstypen und bei der Aufnahme von Forschungsdaten bereit. Durch den Einsatz normierter Metadaten ist eine hohe Interoperabilität genauso gewährleistet wie die leichte Findbarkeit über Suchmaschinen. Um die Nachhaltigkeit zu sichern, werden gängige Metadatenschemata wie MEDS/MODS und DDI verwendet. Als Identifier wird neben der Vergabe von URNs (Uniform Resource Name) ${ }^{7}$ die Zuweisung von DOIs (Digital Object Identifier $)^{8}$ angeboten.

6 Für die Erläuterungen zu D-Space danke ich Dr. Roberto Cozatl, dem Verantwortlichen für Forschungsdatenmanagement an der ULB.

7 Vgl. http://www.dnb.de/DE/Netzpublikationen/URNService/urnservice_node.html.

8 Vgl. https://www.doi.org/. 
D-Space bietet also eine Vielzahl von Metadatenstandards und legt den Schwerpunkt auf Interoperabilität, es werden sowohl OAI-PMH ${ }^{9}$ als auch die SWORD-Protokolle ${ }^{10}$ unterstützt. Das Framework kann so konfiguriert werden, dass es nach bestimmten Archivierungsansätzen arbeitet, z. B. dem OAIS-Referenzmodell. ${ }^{11}$ Weitere Eigenschaften von D-Space, etwa die Unterstützung von Restriktionen bestimmter digitaler Objekte oder Kollektionen und die ItemLevel-Versionierung, erleichtern das Handling der Forschungsdaten.

Durch die D-Space-Anwendungsschicht bietet das System verschiedene Frontend-Module an, von denen aus eine Verbindung zum Hauptsystem aufgebaut wird. Jede Hochschule in Sachsen-Anhalt, die das System nutzen möchte, erhält ein eigenes Frontend. Bei der Anmeldung und der nachfolgenden Identifikation des Benutzerprofils sehen die Benutzer eine vertraute institutsspezifische Informationsseite. Auf dieser können vorab vereinbarte Datentypen hochgeladen und auch vorab vereinbarte Aufgaben durchgeführt werden. Damit sind die Autoren in der Lage, eigene Publikationen und Datensätze selbständig hochzuladen und weiterzubearbeiten. Die Systemadministratoren überprüfen mittels System-Admin-Tools unter anderem die hinzugefügten Einträge und übernehmen damit einhergehende Aufgaben, beispielsweise die Ausgabe von PIDs, ${ }^{12}$ bevor Daten für die Archivierung und die Langzeitarchivierung weiterverarbeitet werden. Sobald die Datenarchivierung stattgefunden hat, ist das System in der Lage, Disseminationsbündel automatisch zum Download und zur Veröffentlichung vorzubereiten. Der Zugriffsumfang auf die Daten wird klar definiert, um die unbefugte Nutzung von Daten durch Personen ohne entsprechende Autorisierung auszuschließen. Durch andere Module des Systems (z. B. METS Exporter) ist es möglich, die Metadaten über REST API ${ }^{13}$ und andere Methoden in zusätzliche Applikationen zu exportieren.

Da die ULB Sachsen-Anhalt über begrenzte personelle Ressourcen verfügt, wird Osiris bedarfsgerecht schrittweise ausgebaut. Die Archivierung von Forschungsdaten erfolgt prototypisch anhand konkreter Projekte. Begonnen wird mit zwei Datenkorpora aus den Bereichen Musikwissenschaft und historische Sozialwissenschaften. Die technische Entwicklung erfolgt in Kooperation mit dem Rechenzentrum der Universität, die funktionellen Anforderungen werden mit den Anwendern zusammen erarbeitet. Der Beirat der wissenschaftlichen Bibliotheken Sachsen-Anhalts begleitet das Projekt und hat den Aufbau beschlos-

\footnotetext{
9 Vgl. https://www.openarchives.org/pmh/.

$10 \mathrm{Vgl}$. https://wiki.duraspace.org/display/DSDOC5x/SWORDv1+Server.

11 Vgl. http://www.oais.info/.

12 PID: Personally-Identifiable Data.

13 Vgl. https://de.wikipedia.org/wiki/Representational_State_Transfer.
} 
sen. Die IT-Kommission des Landes Sachsen-Anhalt unterstützt das Projekt durch Finanzierung der DINI-Zertifizierung. ${ }^{14}$

Das Osiris-Projekt ist auch innerhalb der MLU Teil der Strategie zum Umgang mit Forschungsdaten. Im Rahmen einer Arbeitsgruppe, die wie die OpenAccess-AG beim Prorektorat für Forschung angesiedelt ist, wurde im vierten Quartal 2017 eine Policy zum Umgang mit Forschungsdaten erarbeitet, die aktuell dem Akademischen Senat zur Verabschiedung vorliegt. Die Universität verpflichtet sich, zur Verbesserung der Sichtbarkeit der an der Universität entstandenen Forschungsergebnisse durch Bereitstellung geeigneter Forschungsdateninfrastrukturen beizutragen. Sie verpflichtet sich zudem, die Daten nachhaltig $\mathrm{zu}$ sichern und deren normgerechte Erschließung zu garantieren. Geeignete Schulungs- und Beratungsangebote sollen etabliert werden und fachspezifischen Besonderheiten wird Rechnung getragen.

\section{Sichtbarkeit erhöhen: das Discovery-System}

Die ULB Sachsen-Anhalt betreut mit dem Bibliothekssystem Halle-Merseburg zehn weitere Bibliotheken anderer Hochschulen, außeruniversitärer Einrichtungen oder Stiftungen, z. B. die Bibliothek der Franckeschen Stiftungen, die Marienbibliothek, die Bibliothek der Leopoldina, die Reformationsgeschichtliche Forschungsbibliothek in Wittenberg, die Hochschule Merseburg und weitere. Die Bibliothek der Otto-von-Guericke-Universität Magdeburg betreut im Bibliothekssystem Magdeburg weitere Bibliotheken..$^{15}$ Alle Bibliotheken haben das LBS-System des GBV im Einsatz.

Die Anforderungen der ULB an das einzuführende Discovery-System sind hoch. Zum einen sollte ein möglichst gut ausgestatteter Index zur Verfügung stehen, damit die lizenzierten Volltexte im Direktzugriff möglichst auf Artikelebene recherchierbar und zugreifbar sind. Die ULB selber konnte und wollte keine oder möglichst wenig Personalkapazität für Implementierung und Betreuung zur Verfügung stellen, und es sollte eine Nutzerkontenanbindung existieren, damit mittelfristig die OPACs nicht weiter betrieben werden müssen. Hinzu kommt die Notwendigkeit, die mittlerweile sechsstellige Zahl an digitalisierten Werken in die Suche einzubinden. Auch die in Kalliope oder IKAR erfassten Werke sollen in die Suche integriert werden können. Wünschenswert ist darüber hinaus die Möglichkeit, die umfangreichen Strukturdaten, die in der ULB

14 Vgl. https://dini.de/dini-zertifikat/.

15 Vgl. https://opac.lbs-magdeburg.gbv.de/cgi-bin/wwwopc4menu. 
in den letzten Jahren mit viel Aufwand erstellt worden sind, für die Recherche nutzbar zu machen. Der neue Osiris-Server ist zu integrieren und die unterschiedlichen Datentypen müssen gefunden werden können. In einer letzten Anforderung geht es um die Suchräume, die der Fachinformationsdienst Nahost-, Nordafrika- und Islamstudien (FID) aufbaut. Der FID betreibt aktuell ein eigenes Open-Access-Repositorium, das zukünftig in Osiris eingebunden werden soll. Es gibt umfangreiche Digitalisierungsaktivitäten, die Wissenschaftler können eigene Publikationen einstellen und die Volltexte sind mit Optical Character Recognition (OCR) hinterlegt, sodass sie leicht durchsuchbar sind.

Das Discovery-System soll nicht nur die Anforderungen der ULB und des FID erfüllen, sondern auch für die anderen Bibliotheken des Bibliothekssystems Halle-Merseburg nutzbar sein. Die Hochschulbibliothek Merseburg führt ihre elektronischen Ressourcen mit einem eigenen SFX-Server ${ }^{16}$ und eigener Nutzerverwaltung; die anderen Bibliotheken haben aktuell keine eigene Ressourcenverwaltung.

Nachdem die Entscheidung für das System EDS gefallen ist, werden die genannten Anforderungen schrittweise implementiert und das System geht im ersten Quartal 2018 in Produktion. Als Folge der Umsetzung der Open-AccessStrategie der Universität zeichnen sich weitere Umstrukturierungen ab, die Erwerbungsabteilung und Fachreferate vor neue Herausforderungen stellen.

\section{Open Access, Transformation und Etatverteilung}

Die Einrichtung des Publikationsfonds und die Einführung der freien Open-Access-Projektförderung ziehen mittelfristig eine dauerhafte Umschichtung des Etats nach sich. Zunächst wird der Fonds über frei gewordene Mittel als Folge der DEAL-Verhandlungen und seit 2018 anteilig über die DFG finanziert. Aus Sicht des Etatverteilungsmodells wird der so genannte „Vorabzug“ bemüht.

Die Transformation ${ }^{17}$ der Subskriptionsverträge zum publikationsfinanzierten Open-Access-Lizenzmodell hat jedoch eine systematische Konsequenz. Es muss neu überlegt werden, welche Verteilungsparameter zukünftig die möglichst gerechte Aufteilung der Erwerbungsmittel auf die einzelnen Fächer steuert. Neben die Zahlen der Studierenden und Professoren, des Publikationsaufkommens und der Durchschnittspreise sollten weitere Einflussfaktoren treten.

16 Vgl. https://knowledge.exlibrisgroup.com/SFX.

17 Vgl. z. B. Schimmer, Ralf: „Offsetting“ im Kontext der OA-Transformation, 12. Mai 2017. http://esac-initiative.org/wp-content/uploads/2017/05/2017-05-12_INTACT_Transformation_Offsetting.pdf. 
Denkbar wäre z.B. der prozentuale Anteil an bestehenden Offsetting-Verträgen $^{18}$ oder die jährliche Anzahl an Publikationen, die publikationsfinanziert worden sind und im Open Access zur Verfügung stehen. Berücksichtigt werden sollte dabei, welche Publikationskulturen in den einzelnen Fächern existieren. Es müsste zudem darauf geachtet werden, dass es keine durch das Modell indizierten, sich selbst perpetuierenden Finanzierungstendenzen gibt, die dazu führen, dass bestimmte Fächer, die noch keinen sehr hohen Anteil an Open-Access-Publikationen aufweisen können, in jedem Jahr weniger Mittel zur Verfügung gestellt bekommen. Anreize für den Abschluss von Transformationsverträgen sollten hingegen fester Bestandteil des Modells sein.

Meines Erachtens kommt ein weiterer Faktor hinzu. Die großen Anbieter der Portfolios elektronischer Zeitschriften stellen ihr Verlagsangebot zunehmend auf so genannte „Datenbankmodelle“ um. In der Ausgestaltung variieren die Angebote etwas, aber die Bibliotheken lizenzieren entweder das gesamte Zeitschriftenangebot als ein Paket oder aber sie erwerben mit dem Zugriff auf einzelne Zeitschriften oder Fächerpakete einen Nachlass oder eine kostenfreie Zugabe eines großen Bestands weiterer Zeitschriften. Die somit lizenzierten Gesamtpakete können zwar mit einigem Aufwand auf die Fächer verteilt und anschließend kann die Finanzierung zwischen den Fächern aufgeteilt werden, was aber nur bedingt sinnvoll erscheint. Verhandelt werden die Lizenzen zentral, meist auf regionaler Ebene, in Zukunft hoffentlich oft auf nationaler Ebene. Es werden Bestandteile lizenziert, die nicht unbedingt die Ausstattung eines Faches an der eigenen Universität abbilden und die Wichtigkeit der lizenzierten Inhalte ist sehr unterschiedlich, vor allem, wenn als Zusatzkriterium die OpenAccess-Komponente bewertet wird.

Im Bereich der FID-Lizenzen wird nicht mehr nur die eigene Einrichtung, sondern die Fachcommunity versorgt. Hier werden Lizenzmodelle entwickelt, aber erst die Zugriffe werden in den nächsten Jahren zeigen, ob die Höhe der investierten Mittel angemessen ist. Der DFG-Anteil von 85 \% der Lizenzkosten federt diesen Zeitraum ab, gleichwohl sollten die Lizenzmodelle und das Nutzerklientel im Etatverteilungsmodell berücksichtigt werden. ${ }^{19}$ Die Bibliothekskommission der MLU unterstützt die Umwidmung von Erwerbungsmitteln in Richtung Open Access. Sie wies aber in der letzten Sitzung darauf hin, dass mittelfristig das Etatverteilungsmodell anders ausgestaltet werden sollte: Die ver-

18 Vgl. z. B. http://oa2020-de.org/Offsetting-und-Transformation.

19 Vgl. z.B. Hillenkötter, Kristine: An der Schwelle zur Transformation: „Alte“ und „neue“ Lizenzmodelle im Vergleich, 22. Dezember 2017 [Preprint der Zeitschrift Bibliothek - Forschung und Praxis]. https://edoc.hu-berlin.de/handle/18452/19351. 
änderten Finanzierungswege müssen so im Modell verankert werden, dass kein Bereich benachteiligt wird.

Für die Erwerbungsabteilung ist es in den nächsten Jahren wichtig, nicht allein die DEAL-Vertragsabschlüsse durchzuführen, sondern in die Verhandlungen mit Anbietern Offsetting-Anteile hineinzubringen. Bewertet werden muss etwa die Mitgliedschaft in Vereinen oder bei Herausgebergruppen, die dazu führen, dass Article Processing Charges dieser Zeitschriften preiswerter sind. Manchmal werden vorab Kontingente eingekauft, bei denen im Vorfeld nicht ganz klar ist, welchem Fach der dann publizierte Artikel zugeordnet werden muss. Auch Mitgliedsbeiträge wie z. B. die Mitgliedschaft in der Open Library of Humanities, ${ }^{20}$ einem Verlag für Open-Access-Zeitschriften in den Humanities, sind zu berücksichtigen. Es wäre nicht sinnvoll, solche Kosten im Haushalt über Mitgliedschaften zu planen, denn sie gehören meines Erachtens zum Literaturerwerb und sollten in Zukunft bei der Etatverteilung bedacht werden.

In der Summe bedeuten die Umgestaltung der Lizenzierung, die Unterstützung von Open Access und die Betreuung der Erschließung und Speicherung von Forschungsdaten veränderte Workflows, Lernprozesse und viel Bereitschaft zur Veränderung. Einigen Mitarbeitern fällt es schwer, sich auf diese permanenten Veränderungen einzulassen. Sie sind jedoch oft zugleich die Wissensträger, die die Arbeitsabläufe entwickelt haben und Vor- und Nachteile im Detail kennen. Die Umgestaltung der internen Geschäftsprozesse wird gelingen, wenn das vorhandene Wissen genutzt und wenn gemeinsam bedarfsorientiert im Dialog mit allen Beteiligten gearbeitet wird. Nur so lässt sich verhindern, dass die Belegschaft aufgeteilt wird in einen Teil, der Veränderung initiiert und gestaltet, und in einen, der die Arbeitsabläufe unverändert weiterführt.

\section{Fazit}

Mit den Verankerungen der Open-Access-Strategie und der ForschungsdatenPolicy der MLU, dem neuen Schulungskonzept, dem Publikationsfonds, dem Osiris-Landesserver für Forschungsdaten und Open-Access-Publikationen sowie der Verbesserung der Recherche von frei zugänglichem Content durch Einführung des Discovery-Systems sind die Aktivitäten der ULB Sachsen-Anhalt in Bezug auf Open Science kurz zusammengefasst. Wichtig für die Bibliothek ist die Orientierung an den Bedarfen aller Nutzer dieser Services und die gemeinsame Entwicklung dieser Angebote mit dem Rechenzentrum der Universität und - wo

20 https://www.openlibhums.org/. 
es möglich ist - auch mit dem LLZ-Zentrum für multimediales Lehren und Lernen. Die Zusammenarbeit mit den Bibliotheken über den Beirat für wissenschaftliche Bibliotheken des Landes Sachsen-Anhalt und der fachliche Austausch mit der IT-Kommission des Landes sichern eine gemeinsame Weiterentwicklung der Angebote. Für die Mitarbeiter der Bibliothek bedeuten diese neuen Entwicklungen jedoch eine zusätzliche Arbeitsbelastung. Die Fachreferenten sollen zu vielen neuen Fragestellungen kompetent Auskunft geben können und die zusätzlichen Schulungsangebote kommen zu den bisherigen Programmen hinzu. Inhaltliche Abstimmungen, die über die eigene Bibliothek hinausgehen, sind sehr zeitintensiv, und es macht sich zunehmend deutlich bemerkbar, dass die Bibliothek im Vergleich zu Bibliotheken ähnlicher Größe und Aufgabenstellung mit deutlich weniger Personal ausgestattet ist. 